Betsabé Pérez Garrido - Szabolcs Szilárd Sebrek - Viktoriia Semenova

\title{
Comparing different ranking functions for solving fuzzy linear programming problems with fuzzy cost coefficients*
}

\author{
BETSABÉ PÉREZ GARRIDO (corresponding author) \\ Associate Professor \\ Corvinus University of Budapest, \\ Hungary \\ Email: perez.betsabe@uni-corvinus.hu \\ SZABOLCS SZILÁRD SEBREK \\ Associate Professor \\ Corvinus University of Budapest, \\ Hungary \\ Email: sebrek@uni-corvinus.hu
}

\author{
VIKTORIIA SEMENOVA \\ $\mathrm{PhD}$ Candidate \\ Doctoral School of Business and \\ Management \\ Corvinus University of Budapest, \\ Hungary \\ Email: viktoriia.semenova@stud.uni- \\ corvinus.hu
}

In many applications of linear programming, the lack of exact information results in various problems. Nevertheless, these types of problems can be handled using fuzzy linear programming. This study aims to compare different ranking functions for solving fuzzy linear programming problems in which the coefficients of the objective function (the cost vector) are fuzzy numbers. A numerical example is introduced from the field of tourism and then solved using five ranking functions. Computations were carried out using the FuzzyLP package implemented in the statistical software R.

KEYWORDS: fuzzy linear programing, fuzzy sets, ranking functions

* Szabolcs Szilárd Sebrek and Viktoriia Semenova would like to express their gratitude to the project titled 'From Talent to Young Researcher project aimed at activities supporting the research career model in higher education' IDN. EFOP-3.6.3-VEKOP-16-2017-00007, co-supported by the European Union, Hungary, and the European Social Fund. Viktoriia Semenova would like to thank the National Bank of Hungary for the financial support under the Research Excellence Award. Szabolcs Szilárd Sebrek and Betsabé Pérez Garrido are obliged to the Project No. TKP2020-NKA-02 that has been implemented with the support provided from the National Research, Development, and Innovation Fund of Hungary, financed under the Thematic Excellence Programme funding scheme.

HUNGARIAN STATISTICAL REVIEW, VOLUME 4, NUMBER 2, PP. 3-17. DOI: 10.35618/hsr2021.02.en003 
Linear programming (LP) is one of the most widely used mathematical techniques for solving real-world problems in which the goal is to maximize (or minimize) a linear objective function subject to a finite number of linear constraints. These problems are characterized by a panoply of possible solutions that satisfy a set of linear constraints. The selection of the optimal solution depends on the specific goal of the problem defined in the objective (or cost) function. A large body of literature has illustrated its applicability, such as in the agricultural sector (Alotaibi-Nadeem [2021]), production processes (Krynke-Mielczarek [2018]), the field of energy (PolatGürtuna [2018]), and transportation (Patel-Patel-Bhathawala [2017]).

Scholars have placed a special interest in developing a flexible approach for handling imprecise, inaccurate, or vague information presented in LP problems. Fuzzy linear programming has emerged and gained significant interest (Ghanbari et al. [2020], Saati et al. [2015], Cardenas-Verdegay [1997]). Following this line of research, we aim to provide a comparative analysis for solving fuzzy linear programming problems with fuzzy cost coefficients using different ranking functions.

The study is organised as follows: after having given an overview of the classical LP problem in Section 1, Section 2 provides an introduction of fuzzy theory. Section 3 discusses fuzzy linear programming and Section 4 then addresses ranking functions. Next, Section 5 examines a numerical example from the field of tourism. The work ends with a conclusion in Section 6, summarising the main findings and giving recommendations to practitioners.

\section{Classical LP problem}

The classical (or crisp) LP problem aims to maximize (or minimize) a linear objective function subject to a finite number of linear constraints. Without loss of generality, the general LP problem can be formulated as follows (NasseriEbrahimnejad-Cao [2019]):

$$
\begin{gathered}
\max \mathbf{z}=\mathbf{c x} \\
\text { s.t. } \mathbf{A x} \leq \mathbf{b} \\
\mathbf{x} \geq 0
\end{gathered}
$$


where $\mathbf{c}^{T}=\left(c_{1}, \ldots, c_{n}\right)^{T} \in \mathbb{R}^{n}$ is the cost vector, $\mathbf{x}=\left(x_{1}, \ldots, x_{n}\right)^{T} \in \mathbb{R}^{n}$ is the vector of decision variables, $\mathbf{b}=\left(b_{1}, \ldots, b_{m}\right)^{T} \in \mathbb{R}^{m}$ is the vector of coefficients (right-hand side of the constraints), and $\mathbf{A}=\left[a_{i j}\right]_{m \times n} \in \mathbb{R}^{m \times n}$ is the coefficient matrix (lefthand side of the constraints).

In this context, given a cost vector $\mathbf{c}^{T}=\left(c_{1}, \ldots, c_{n}\right)^{T}$, the goal is to maximize (or minimize) the linear objective function $\mathbf{c x}=\sum_{i=1}^{n} c_{i} x_{i}$ subject to a finite number of linear constraints. A relevant disadvantage attached to this approach is that it assumes the researcher to have an exact knowledge of the model under consideration the exact coefficients of the cost vector (c), left-hand side of the constraints (A), and right-hand side of the constraints (b). It also assumes no ambiguity (or uncertainty) in the vector of decision variables, $\mathbf{x}$.

However, in many real applications, obtaining the required information may involve a considerable amount of time and effort. Indeed, it is not always possible to obtain the exact information but instead information with a high degree of imprecision (or fuzziness). Fuzzy theory provides a mathematical framework to model quantities that are imprecise, inaccurate, or vague. The combination of fuzzy theory and LP has emerged under the name 'fuzzy linear programming' (FLP) (Zimmermann [1978], Bector-Chandra [2005], Nasseri-Ebrahimnejad-Cao [2019], Fang et al. [1999]). Thus, FLP provides a mathematical framework for approaching imprecise (fuzzy) information presented in many LP applications.

\section{Preliminaries: fuzzy theory}

Definition 1 (Fuzzy set and membership function). Let $X$ be the universe whose generic element is denoted by $x$. A fuzzy set $\widetilde{C}$ is a function, $\widetilde{C}: X \rightarrow[0,1]$ that can be represented by a set of ordered pairs:

$$
\widetilde{C}=\left\{\left(x, \mu_{\widetilde{C}}(x)\right) \mid x \in X\right\}
$$

where $\mu_{\widetilde{C}}(\cdot)$ is called the membership function of the fuzzy set. 
The membership function represents the strength with which $x$ is contained in $\widetilde{C}$. Intuitively, it can be interpreted as follows:

- If the membership degree of $x$ is equal to 1 , then value $x$ definitely belongs to the fuzzy set.

- If the membership degree of $x$ is equal to 0 , then value $x$ definitely does not belong to the fuzzy set.

- If the membership function belongs to $[0,1]$, then it can be defined in several ways (e.g. representing a trapezoidal or triangular form).

In contrast, the membership function of the classical LP approach (see Section 1) can be defined using only two elements:

$$
\mu_{C}(x)=\left\{\begin{array}{l}
0, \text { if } x \in C \\
1, \text { if } x \notin C
\end{array} .\right.
$$

Definition 2 (Support and core of a fuzzy set). The support of fuzzy set $\widetilde{C}$ is the set of all $x \in X$ with $\mu_{\widetilde{C}}(x)>0$. The core of fuzzy set $\widetilde{C}$ is the set of all $x \in X$ with $\mu_{\widetilde{C}}(x)=1$.

Definition 3 (Trapezoidal and triangular fuzzy numbers). Let $\tilde{a}=\left(a^{L}, a^{R}, L, R\right)$ with $L<a^{L}<a^{R}<R$ being real numbers. Fuzzy number $\tilde{a}$ is called trapezoidal fuzzy number (TrFN) if its membership function is:

$$
\mu_{\tilde{a}}(x)=\left\{\begin{array}{cl}
\frac{x-L}{a^{L}-L} & \text { if } L \leq x \leq a^{L} \\
1 & \text { if } a^{L} \leq x \leq a^{R} \\
\frac{R-x}{R-a^{R}} & \text { if } a^{R} \leq x \leq R \\
0 & \text { otherwise }
\end{array} .\right.
$$

HungaRian STATISTICAL REVIEW, VOLUME 4, NuMBER 2, PP. 3-17. DOI: 10.35618/hsr2021.02.en003 
Figure 1. Graphical representation of a $\operatorname{TrFN}$

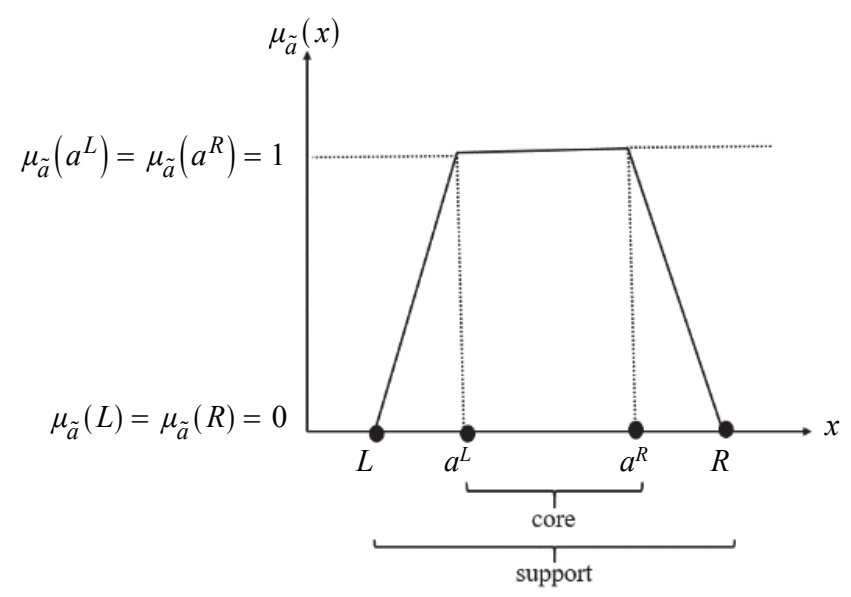

A TrFN holds the following properties:

$-\mu_{\tilde{a}}(x)$ is non-decreasing on range $\left[L, a^{L}\right]$.

$-\mu_{\tilde{a}}(x)$ is non-increasing on range $\left[a^{R}, R\right]$.

- The support is defined in the interval $[L, R]$.

- The core is defined in the interval $\left[a^{L}, a^{R}\right]$.

$-\mu_{\tilde{a}}(x)=0$ for all $x \in(-\infty, L]$ and all $x \in[R, \infty)$.

Particularly, if $a^{L}=a^{R}=a$, we obtain a triangular fuzzy number (TFN) denoted as $\tilde{a}=(a, L, R)$.

\section{Fuzzy linear programming}

Uncertainty (or fuzziness) in LP problems may occur in different ways. Depending on the source of this uncertainty, FLP problems can be classified in different groups. (See Table 1.)

In the present work, we focus on the solution for the first group (FLP problems with fuzzy cost coefficients) using ranking functions. The following section introduces several ranking functions.

Hungarian Statistical ReVIEW, VOlume 4, NuMBER 2, PP. 3-17. DOI: 10.35618/hsr2021.02.en003 
Classification of FLP problems

\begin{tabular}{|c|c|}
\hline Group & Mathematical model \\
\hline Group 1. FLP problems with fuzzy cost coefficients, $\tilde{\mathbf{c}}$. & $\begin{array}{l}\max \tilde{\mathbf{z}} \approx \tilde{\mathbf{c} x} \\
\text { s.t. } \mathbf{A x} \leq \mathbf{b} \\
\quad \mathbf{x} \geq 0\end{array}$ \\
\hline $\begin{array}{l}\text { Group } 2 . \text { FLP problems with fuzzy numbers for the coefficients of the decision variables in } \\
\text { the constraints and the right-hand side of the constraints, } \tilde{\mathbf{A}} \text { and } \tilde{\mathbf{b}} \text {, respectively. }\end{array}$ & $\begin{array}{l}\max \mathbf{z}=\mathbf{c x} \\
\text { s.t. } \tilde{\mathbf{A}} \mathbf{x} \leq \tilde{\mathbf{b}} \\
\quad \mathbf{x} \geq 0\end{array}$ \\
\hline $\begin{array}{l}\text { Group 3. FLP problems with fuzzy cost coefficients, } \tilde{\mathbf{c}} \text {. Fuzzy numbers for the coefficients } \\
\text { of the decision variables in the constraints and the right-hand side of the constraints, } \\
\tilde{\mathbf{A}} \text { and } \tilde{\mathbf{b}} \text {, respectively. }\end{array}$ & $\begin{array}{l}\max \tilde{\mathbf{z}} \approx \tilde{\mathbf{c}} \mathbf{x} \\
\text { s.t. } \tilde{\mathbf{A}} \mathbf{x} \leq \tilde{\mathbf{b}} \\
\quad \mathbf{x} \geq 0\end{array}$ \\
\hline $\begin{array}{l}\text { Group } 4 \text {. FLP problems with fuzzy numbers for the decision variables, } \tilde{\mathbf{x}} \text {. Fuzzy numbers } \\
\text { for the coefficients of the right-hand side of the constraints, } \tilde{\mathbf{b}} \text {. }\end{array}$ & $\begin{array}{l}\max \tilde{\mathbf{z}} \approx \mathbf{c} \tilde{\mathbf{x}} \\
\text { s.t. } \mathbf{A} \tilde{\mathbf{x}} \leq \tilde{\mathbf{b}} \\
\tilde{\mathbf{x}} \geq \tilde{0}\end{array}$ \\
\hline $\begin{array}{l}\text { Group 5. FLP problems with fuzzy cost coefficients, } \tilde{\mathbf{c}} \text {. Fuzzy numbers for the decision } \\
\text { variables, } \tilde{\mathbf{x}} \text {. Fuzzy numbers for the coefficients of the right-hand side of the constraints, } \tilde{\mathbf{b}} \text {. }\end{array}$ & $\begin{array}{l}\max \tilde{\mathbf{z}} \approx \tilde{\mathbf{c}} \tilde{\mathbf{x}} \\
\text { s.t. } \mathbf{A} \tilde{\mathbf{x}} \leq \tilde{\mathbf{b}} \\
\tilde{\mathbf{x}} \geq \tilde{0}\end{array}$ \\
\hline $\begin{array}{l}\text { Group 6. FLP problems with fuzzy numbers for the decision variables, } \tilde{\mathbf{x}} \text {. Fuzzy numbers } \\
\text { for the coefficients of the decision variables in the constraints and the right-hand side of the } \\
\text { constraints, } \tilde{\mathbf{A}} \text { and } \tilde{\mathbf{b}} \text {, respectively. }\end{array}$ & $\begin{array}{l}\max \tilde{\mathbf{z}} \approx \mathbf{c} \tilde{\mathbf{x}} \\
\text { s.t. } \tilde{\mathbf{A}} \tilde{\mathbf{x}} \leq \tilde{\mathbf{b}} \\
\tilde{\mathbf{x}} \geq \tilde{0}\end{array}$ \\
\hline $\begin{array}{l}\text { Group } 7 \text {. FLP is fully fuzzy, involving fuzzy cost coefficients, } \tilde{\mathbf{c}} \text {. Fuzzy numbers in the } \\
\text { decision variables, } \tilde{\mathbf{x}} \text {. Fuzzy numbers for the coefficients of the decision variables in the } \\
\text { constraints and the right-hand side of the constraints, } \tilde{\mathbf{A}} \text { and } \tilde{\mathbf{b}} \text {, respectively. }\end{array}$ & $\begin{array}{l}\max \tilde{\mathbf{z}} \approx \tilde{\mathbf{c}} \tilde{\mathbf{x}} \\
\text { s.t. } \tilde{\mathbf{A}} \tilde{\mathbf{x}} \leq \tilde{\mathbf{b}} \\
\quad \tilde{\mathbf{x}} \geq \tilde{0}\end{array}$ \\
\hline
\end{tabular}

Note. In this table, we consider the following fuzzy notations: $\tilde{\mathbf{c}}^{T}=\left(\tilde{c}_{1}, \ldots, \tilde{c}_{n}\right)^{T}, \quad \tilde{\mathbf{b}}=\left(\tilde{b}_{1}, \ldots, \tilde{b}_{m}\right)^{T}$, $\tilde{\mathbf{x}}=\left(\tilde{x}_{1}, \ldots, \tilde{x}_{n}\right)^{T}$, and $\tilde{\mathbf{A}}=\left[\tilde{a}_{i j}\right]_{m \times n}$, with $\tilde{c}_{j}, \tilde{b}_{i}, \tilde{x}_{j}, \tilde{a}_{i j}$ being fuzzy numbers, for $i=1, \ldots, m, j=1, \ldots, n$.

Source: Saati et al. [2015].

\section{Ranking functions}

For the FLP problem with fuzzy cost coefficients (Group 1 of Table 1), the FLP problem can be written as follows:

$$
\max \tilde{\mathbf{z}} \approx \tilde{\mathbf{c}} \mathbf{x}
$$




$$
\begin{gathered}
\text { s.t. } \mathbf{A x} \leq \mathbf{b} \\
\mathbf{x} \geq 0
\end{gathered}
$$

where $\tilde{\mathbf{c}}^{T}=\left(\tilde{c}_{1}, \ldots, \tilde{c}_{n}\right)^{T}$, and $\tilde{c}_{j}$ being fuzzy numbers for $j=1, \ldots, n$.

The solution of (2) can be carried out by converting the original problem into its equivalent crisp problem and then using standard methods (NasseriEbrahimnejad-Cao [2019]). The transformation of a fuzzy problem into a crisp problem can be achieved using the concept of ranking function. A ranking function maps each fuzzy number into the real line, $\mathbb{R}$,

$$
R=F(R) \rightarrow \mathbb{R}
$$

Ranking functions are especially useful for comparing fuzzy numbers. In general, comparing fuzzy numbers can be extremely difficult (McCahon-Lee [1990]). If $\tilde{a}$ and $\tilde{b}$ are two fuzzy numbers, then both numbers can be compared using the following:

$$
\begin{aligned}
& \tilde{a}>\tilde{b} \Leftrightarrow R(\tilde{a})>R(\tilde{b}) \\
& \tilde{a}<\tilde{b} \Leftrightarrow R(\tilde{a})<R(\tilde{b}) \\
& \tilde{a} \approx \tilde{b} \Leftrightarrow R(\tilde{a})=R(\tilde{b})
\end{aligned}
$$

where $R$ denotes a ranking function.

Moreover, if the ranking function satisfies the condition $R(k \cdot \tilde{a}+\tilde{b})=k \cdot R(\tilde{a})+R(\tilde{b})$ for any two fuzzy numbers $\tilde{a}$ and $\tilde{b}$ and any $k \in \mathbb{R}$, then it is called a linear ranking function. To solve problem (2), we can transform it into an equivalent crisp problem, replacing its fuzzy cost function with

$$
\max \mathbf{z}=R\left(\tilde{c}_{1}\right) x_{1}+\ldots,+R\left(\tilde{c}_{n}\right) x_{n}
$$

where $R$ denotes a ranking function. 
Five ranking functions proposed in the literature

\begin{tabular}{|c|c|c|c|}
\hline No. & Author & Function's name & Ranking function for $\operatorname{TrFN}$ \\
\hline 1 & Yager [1978] & Yager's $F_{1}$ & $R(\tilde{a})=\frac{1}{3} \cdot \frac{\left[\left(a^{R}\right)^{2}-\left(a^{L}\right)^{2}\right]+\left(R^{2}-L^{2}\right)+\left(R a^{R}-L a^{L}\right)}{\left(a^{R}-a^{L}\right)+(R-L)}$ \\
\hline 2 & Yager [1978] & Yager's $F_{3}$ & $R(\tilde{a})=\frac{L+a^{L}+a^{R}+R}{4}$ \\
\hline 3 & Adamo [1980] & Adamo & $R(\tilde{a})=R-\alpha \cdot\left(R-a^{R}\right)$ for fixed $\alpha \in[0,1]$ \\
\hline 4 & $\begin{array}{l}\text { Campos-González } \\
{[1989]}\end{array}$ & Campos & $\begin{array}{l}\qquad R(\tilde{a})=a^{L}+\lambda \cdot\left[\left(a^{R}-a^{L}\right)+\frac{L+R}{3}\right]-\frac{L}{3} \\
\text { The parameter } \lambda \in[0,1] \text { may be interpreted as an optimist/ } \\
\text { pessimist degree which must be selected by the decision maker. } \\
\text { An optimist choice is indicated by values close to } 1 \text {, while the } \\
\text { pessimist choice is indicated by values close to } 0 \text {. }\end{array}$ \\
\hline 5 & González [1990] & González & $\begin{array}{l}\quad R(\tilde{a})=a^{L}-\frac{a^{L}-L}{t+1}+\lambda \cdot\left[a^{R}-a^{L}+\frac{(R-L)-\left(a^{R}-a^{L}\right)}{t+1}\right], \\
\qquad \lambda \in[0,1], t \geq 0 \\
\lambda \text { has the same definition as in Campos's function. }\end{array}$ \\
\hline
\end{tabular}

\section{Numerical example: estimating the maximum revenue generated by tourists in Venice}

This section considers a recent real-world problem introduced in the field of tourism (Bertocchi et al. [2020]). The goal is to maximize the economic benefit generated by tourists in the Italian city of Venice under several socio-environmental constraints. The original FLP problem was formulated as follows:

$$
\begin{gathered}
\max \tilde{\mathbf{z}} \approx \tilde{\mathbf{c}} \mathbf{x} \\
\text { s.t. } \tilde{\mathbf{A}} \mathbf{x} \leq \tilde{\mathbf{b}} \\
\mathbf{x} \geq 0
\end{gathered}
$$


where $\mathbf{x}=(\mathrm{TH}, \mathrm{NTH}, \mathrm{E})^{T} \in \mathbb{R}^{3}$ is the vector of decision variables representing the type of tourists: TH (tourist in hotels), NTH (tourist in other types of accommodations), and $\mathrm{E}$ (excursionist or day tourist).

The cost vector $\tilde{\mathbf{c}}^{T}=\left(\tilde{c}_{1}, \tilde{c}_{2}, \tilde{c}_{3}\right)^{T}$ represents the economic benefit per day generated by each type of tourist stated as uncertain. To capture the uncertainty in these values, we defined the cost vector using the fuzzy numbers $\tilde{c}_{1}=(190,210,230), \tilde{c}_{2}=(140,160,180)$, and $\tilde{c}_{3}=(45,60,80)$. The constraints reflect the most frequently used tourism subsystems (and their maximum capacity) in the historical centre of Venice. This information is represented by

$$
\tilde{\mathbf{A}} \mathbf{x}=\left(\begin{array}{ccc}
\mathrm{TH} & \mathrm{NT} & \mathrm{E} \\
a_{11} & a_{12} & a_{13} \\
a_{21} & a_{22} & a_{23} \\
\tilde{a}_{31} & \tilde{a}_{32} & a_{33} \\
\tilde{a}_{41} & \tilde{a}_{42} & a_{43} \\
\tilde{a}_{51} & \tilde{a}_{52} & a_{53} \\
\tilde{a}_{61} & \tilde{a}_{62} & a_{63} \\
\tilde{a}_{71} & \tilde{a}_{72} & a_{73}
\end{array}\right) \cdot\left(\begin{array}{c}
x_{1} \\
x_{2} \\
x_{3}
\end{array}\right) \quad \text { and } \quad \tilde{\mathbf{b}}=\left(\begin{array}{c}
\tilde{b}_{1} \\
\tilde{b}_{2} \\
\tilde{b}_{3} \\
\tilde{b}_{4} \\
\tilde{b}_{5} \\
\tilde{b}_{6} \\
\tilde{b}_{7}
\end{array}\right) .
$$

The first constraint corresponds to the number of beds in hotels (from one- to five-star hotels), where $\left(a_{11}, a_{12}, a_{13}\right)=(1,0,0)$ and $\tilde{b}_{1}$ is a fuzzy number representing the uncertainty in maximum capacity, defined as $\tilde{b}_{1}=(13,000 ; 14,400 ; 15,500)$. The second constraint is the number of beds in non-hotels, where $\left(a_{21}, a_{22}, a_{23}\right)=(0,1,0)$, and, similarly, $\tilde{b}_{2}$ is a fuzzy number representing the uncertainty in maximum capacity, defined as $\tilde{b}_{2}=(18,500 ; 20,400 ; 22,000)$. The third constraint refers to the capacity (number of seats) in restaurants, pizzerias, and dining rooms. The occupancy by type of tourist is indicated by the fuzzy numbers $\left(\tilde{a}_{31}, \tilde{a}_{32}, \tilde{a}_{33}\right)=[(0.675,0.750,0.825),(0.585,0.650,0.715),(0.180,0.200,0.220)]$, and maximum capacity is shown by the fuzzy number $\tilde{b}_{3}=(23,300 ; 24,000 ; 24,700)$. The fourth constraint is related to the number of available parking spaces. The occupancy by type of tourist is specified by the fuzzy numbers $\left(\tilde{a}_{41}, \tilde{a}_{42}, \tilde{a}_{43}\right)=[(0.297,0.330,0.363),(0.297,0.330,0.363),(0.675,0.750,0.825)]$, and maximum capacity is defined as $\tilde{b}_{4}=(19,000 ; 20,000 ; 21,000)$. The fifth constraint corresponds to the capacity of public transportation, where 
$\left(\tilde{a}_{51}, \tilde{a}_{52}, \tilde{a}_{53}\right)=[(0.9,1.0,1.1),(0.9,1.0,1.1),(0.9,1.0,1.1)]$, and maximum capacity is reflected by the fuzzy number $\tilde{b}_{5}=(45,000 ; 46,000 ; 47,000)$. The sixth constraint is the daily quantity of waste production (in $\mathrm{kg}$ ) imputable to tourists. The quantity for the three types of tourists is represented by the fuzzy numbers $\left(\tilde{a}_{61}, \tilde{a}_{62}, \tilde{a}_{63}\right)=[(2.1,2.3,2.5),(1.8,2.0,2.2),(0.9,1.0,1.1)]$, and maximum capacity is defined by the fuzzy number $\tilde{b}_{6}=(90,000 ; 100,000 ; 110,000)$. The last constraint is associated with the maximum number of people who can enter St Mark's Square for security reasons. The entrance by type of tourist is specified by the fuzzy numbers $\left(\tilde{a}_{71}, \tilde{a}_{72}, \tilde{a}_{73}\right)=[(0.3,0.4,0.5),(0.2,0.3,0.4),(0.6,0.7,0.8)]$, and maximum capacity is indicated by the fuzzy number $\tilde{b}_{7}=(90,000 ; 100,000 ; 110,000)$.

In this work, we simplified the original problem considering an FLP problem with fuzzy cost coefficients,

$$
\begin{gathered}
\max \tilde{\mathbf{z}} \approx \tilde{\mathbf{c} x} \\
\text { s.t. } \mathbf{A x} \leq \mathbf{b} \\
\mathbf{x} \geq 0
\end{gathered}
$$

where $\mathbf{x}=(\mathrm{TH}, \mathrm{NTH}, \mathrm{E})^{T} \in \mathbb{R}^{3}$ is the vector of decision variables. The cost vector $\tilde{\mathbf{c}}^{T}=\left(\tilde{c}_{1}, \tilde{c}_{2}, \tilde{c}_{3}\right)^{T}$ is defined using the fuzzy numbers $\tilde{c}_{1}=(190,210,230)$, $\tilde{c}_{2}=(140,160,180)$, and $\tilde{c}_{3}=(45,60,80)$.

Figure 2. Graphical representation of the membership functions of the cost vector
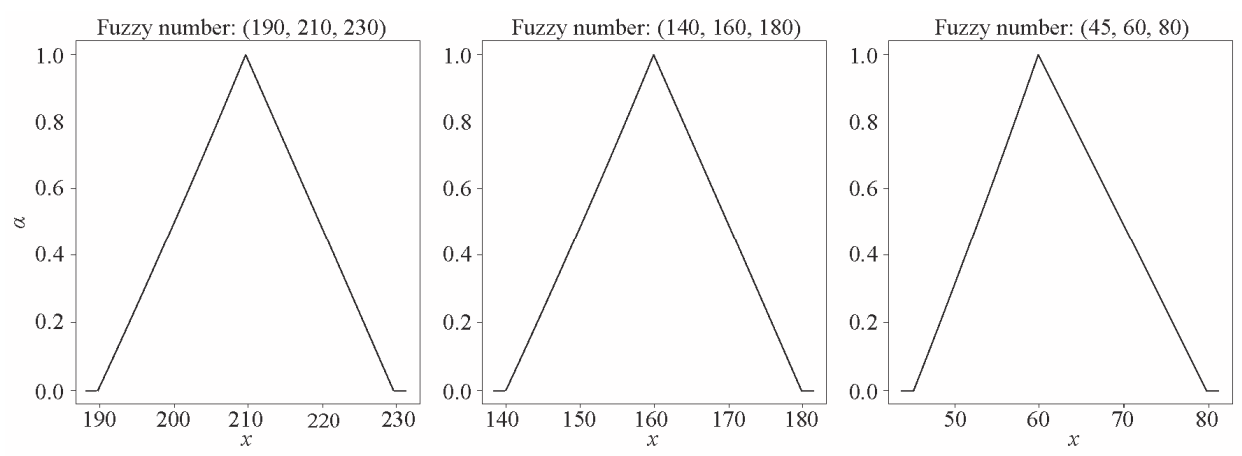

Hungarian StatisticAl REVIEW, VOlume 4, NumBER 2, PP. 3-17. DOI: 10.35618/hsr2021.02.en003 
The constraints are represented as follows:

$$
\tilde{\mathbf{A}} \mathbf{x}=\left(\begin{array}{ccc}
\mathrm{TH} & \mathrm{NT} & \mathrm{E} \\
1 & 0 & 0 \\
0 & 1 & 0 \\
0.75 & 0.65 & 0.2 \\
0.33 & 0.33 & 0.75 \\
1 & 1 & 1 \\
2.3 & 2 & 1 \\
0.4 & 0.3 & 0.7
\end{array}\right) \cdot\left(\begin{array}{l}
x_{1} \\
x_{2} \\
x_{3}
\end{array}\right) \text { and } \tilde{\mathbf{b}}=\left(\begin{array}{c}
14,400 \\
20,400 \\
24,000 \\
20,000 \\
46,000 \\
100,000 \\
100,000
\end{array}\right) .
$$

The FLP problem (3) can be transformed into its equivalent crisp LP problem replacing the fuzzy cost function by

$$
\max \mathbf{z}=R\left(\tilde{c}_{1}\right) x_{1}+R\left(\tilde{c}_{2}\right) x_{2}+R\left(\tilde{c}_{3}\right) x_{3}
$$

where $R$ is one of the ranking functions defined in Table 2 .

Table 3 presents the transformed cost functions using each ranking function from Table 2. The rightmost column displays the optimal values for each decision variable and estimated maximum benefit.

Table 3

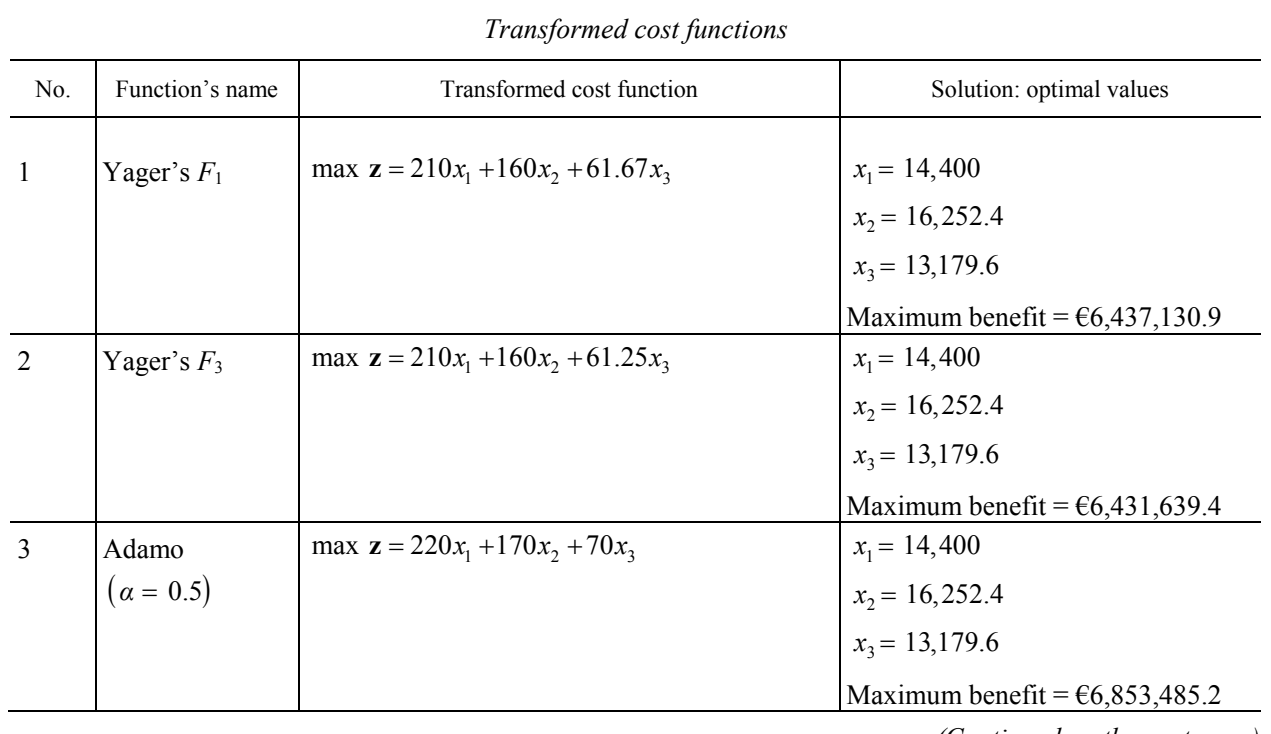

(Continued on the next page) 


\begin{tabular}{|c|c|c|c|}
\hline No. & Function's name & Transformed cost function & Solution: optimal values \\
\hline 4 & $\begin{array}{l}\text { Campos } \\
(\lambda=0.5)\end{array}$ & $\max \mathbf{z}=216.67 x_{1}+113.33 x_{2}+45 x_{3}$ & $\begin{array}{l}x_{1}=14,400 \\
x_{2}=16,252.4 \\
x_{3}=13,179.6 \\
\text { Maximum benefit }=€ 5,555,024.1\end{array}$ \\
\hline 5 & $\begin{array}{l}\text { González } \\
\left(\begin{array}{c}\alpha=0.5 \\
\lambda=0.5\end{array}\right)\end{array}$ & $\max \mathbf{z}=209.57 x_{1}+160 x_{2}+61.67 x_{3}$ & $\begin{array}{l}x_{1}=14,400 \\
x_{2}=16,252.4 \\
x_{3}=13,179.6 \\
\text { Maximum benefit }=€ 6,430,870.0\end{array}$ \\
\hline
\end{tabular}

Note. $x_{1}$ : optimal number of tourists in hotels; $x_{2}$ : optimal number of tourists in other types of accommodations; $x_{3}$ : optimal number of excursionists or day tourists.

Figure 3 gives a graphical representation of the feasible region and optimal values for the vector of decision variables $\mathbf{x}=(\mathrm{TH}, \mathrm{NTH}, \mathrm{E})^{T}$. According to Table 3, in all cases, the optimal number of tourists in the Italian city of Venice (marked with red in Figure 3) is as follows: TH (tourists in hotels) $=14,400, \mathrm{NTH}$ (tourists in other types of accommodations) $=16,252.4$ (rounded to 16,252), and $\mathrm{E}$ (excursionists or day tourists) $=13,179.6$ (rounded to 13,180 ).

Figure 3. Graphical representation of the feasible region and optimal values of vector $\mathbf{x}$

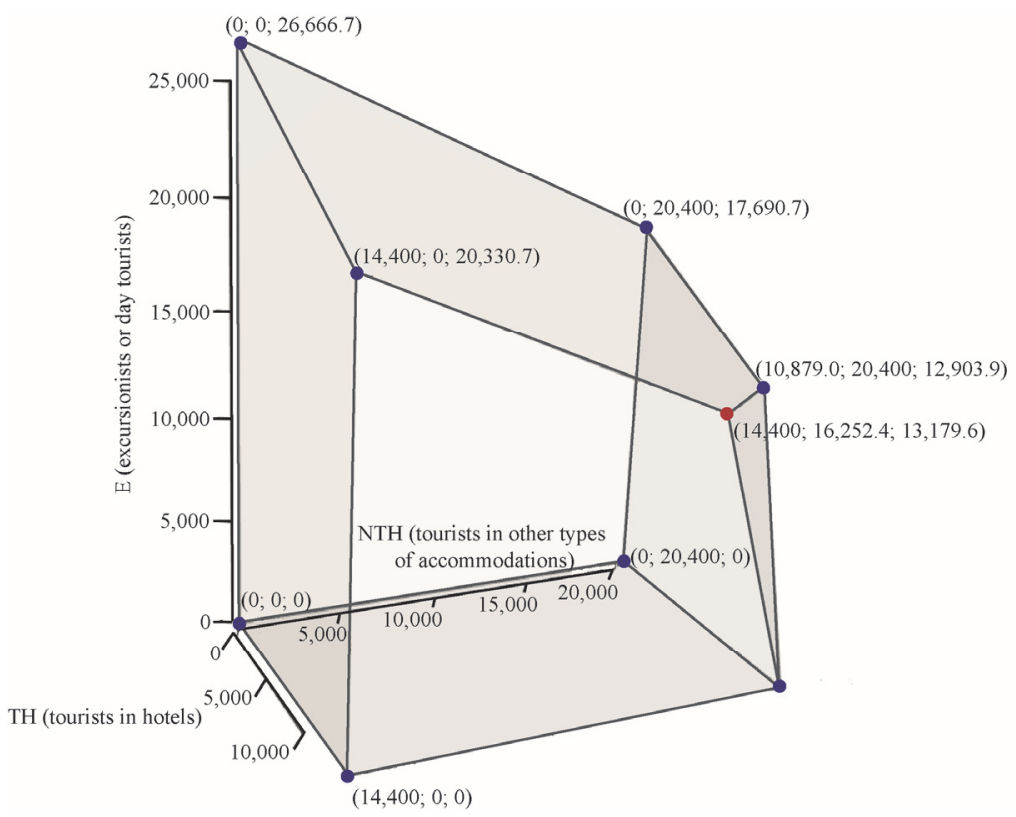

HungaRian STATISTICAL REVIEW, VOLUME 4, NuMBER 2, PP. 3-17. DOI: 10.35618/hsr2021.02.en003 
Given the uncertainty in the economic benefit generated by each tourist type (represented by the fuzzy cost vector $\tilde{\mathbf{c}}^{T}=\left(\tilde{c}_{1}, \tilde{c}_{2}, \tilde{c}_{3}\right)^{T}$ ), the estimation of the maximum benefit will depend on the selected ranking function. Considering the five ranking functions in Tables 2 and 3, the estimated maximum benefit generated by tourists in the Italian city of Venice varies from $€ 5,555,024.1$ to $€ 6,853,485.2$.

\section{Conclusion}

We focused on the solution for FLP problems in which the coefficients of the objective function (cost vector) are fuzzy numbers. The optimal solution was obtained using the ranking function approach, in which the original fuzzy problem is transformed into a crisp problem and then solved using standard methods.

We introduced a numerical example from the field of tourism where the goal was to maximize the economic benefit generated by tourists in the Italian city of Venice subject to different socio-environmental constraints. The benefit generated by each tourist type was assumed to be uncertain and represented by the fuzzy numbers $\tilde{c}_{1}=(190,210,230), \quad \tilde{c}_{2}=(140,160,180)$, and $\tilde{c}_{3}=(45,60,80)$. Based on our results, the optimum number of visitors to Venice per day is 43,832 people, of whom 14,400 are tourists staying in hotels, 16,252 are tourists in other types of accommodations, and 13,180 are one-day tourists.

The estimation of the maximum benefit generated by tourists in Venice depends on the selected ranking function. Considering the five different ranking functions, this quantity varies from $€ 5,555,024.1$ to $€ 6,853,485$.2. Specifically, the estimated maximum benefit using Yager's $F_{1}$ function is $€ 6,437,130.9$; using Yager's $F_{3}$ is $€ 6,431,639.4$; using Adamo function $(\alpha=0.5)$ is $€ 6,853,485.2$; using Campos function $(\lambda=0.5)$ is $€ 5,555,024.1$; and using González function $(\alpha=0.5, \lambda=0.5)$ is $€ 6,430,870$.

Finally, for practitioners, we recommend the use of different ranking functions for developing scenarios, such as a pessimist scenario (in our case, the minimum) and an optimist scenario (in our case, the maximum). From a computational point of view, we suggest the application of the FuzzyLP package implemented in $\mathrm{R}$ (Villacorta et al. [2017], $R$ Core Team [2020]). However, for non-programmers we advise the use of Solver implemented in Excel.

Hungarian Statistical Review, VOlume 4, NumBer 2, PP. 3-17. DOI: 10.35618/hsr2021.02.en003 


\section{References}

Adamo, J. M. [1980]: Fuzzy decision trees. Fuzzy Sets and Systems. Vol. 4. No. 3. pp. 207-219. https://doi.org/10.1016/0165-0114(80)90011-1

AlotAiBI, A. - NADEEM, F. [2021]: A review of applications of linear programming to optimize agricultural solutions. International Journal of Information Engineering and Electronic Business. Vol. 13. No. 2. pp. 11-21. https://doi.org/10.5815/ijieeb.2021.02.02

BeCtor, C. R. - Chandra, S. [2005]: Fuzzy Mathematical Programming and Fuzzy Matrix Games. Studies in Fuzziness and Soft Computing. Springer. Berlin.

Bertocchi, D. - CAmmati, N. - Giove, S. - VAN Der BorG, J. [2020]: Venice and overtourism: Simulating sustainable development scenarios through a tourism carrying capacity model. Sustainability. Vol. 12. No. 2. pp. 512-527. https://doi.org/10.3390/su12020512

CAmpos, L. M. - GonZÁlez, A. [1989]: A subjective approach for ranking fuzzy numbers. Fuzzy Sets and Systems. Vol. 29. No. 2. pp. 145-153. https://doi.org/10.1016/01650114(89)90188-7

CARdenas, J. M. - Verdegay, J. L. [1997]: Using fuzzy numbers in linear programming. IEEE Transactions on Systems, Man and Cybernetics, Part B (Cybernetics). Vol. 27. No. 6. pp. 1016-1022. http://dx.doi.org/10.1109/3477.650062

FANG, S. C. - Hu, C. F. - WANG, H. F. - WU, S. Y. [1999]: Linear programming with fuzzy coefficients in constraints. Computers and Mathematics with Applications. Vol. 37. No. 10. pp. 63-76. https://doi.org/10.1016/S0898-1221(99)00126-1

Ghanbari, R. - Ghorbani-Moghadam, K. - Mahdavi-Amiri, N. - De Baets, B. [2020]: Fuzzy linear programming problems: Models and solutions. Soft Computing. Vol. 24. November. pp. 10043-10073. https://doi.org/10.1007/s00500-019-04519-w

GONZÁLEZ, A. [1990]: A study of the ranking function approach through mean values. Fuzzy Sets and Systems. Vol. 35. No. 1. pp. 29-41. https://doi.org/10.1016/0165-0114(90)90016-Y

KRYNKE, M. - MielCZAREK, K. [2018]: Applications of linear programming to optimize the costbenefit criterion in production processes. MATEC Web of Conferences. Vol. 183. Article No. 04004. http://dx.doi.org/10.1051/matecconf/201818304004

MCCAHON, C. S. - LeE, E. S. [1990]: Comparing fuzzy numbers: The proportion of the optimum method. International Journal of Approximate Reasoning. Vol. 4. No. 3. pp. 159-181. https://doi.org/10.1016/0888-613X(90)90019-X

NAsSERI, S. H. - EbrahimnejAd, A. - CAO, B. Y. [2019]: Fuzzy linear programming: Solution techniques and applications. Studies in Fuzziness and Soft Computing. Vol. 379. Springer. Cham. https://doi.org/10.1007/978-3-030-17421-7_2

Patel, R. G. - Patel, B. S. - Bhathawala, P. H. [2017]: On optimal solution of a transportation problem. Global Journal of Pure and Applied Mathematics. Vol. 13. No. 9. pp. 6201-6208.

PolAt, U. - GÜRTUNA, F. [2018]: A review of applications of linear programming and mixed integer linear programming in energy management: From policy makers/producers to consumers. European Journal of Engineering and Applied Sciences. Vol. 1. No. 2. pp. 84-89.

R Core TeAm [2020]: $R$ : A Language and Environment for Statistical Computing. R Foundation for Statistical Computing. Vienna. http://www.R-project.org/

HungaRian StATISTICAL REVIEW, VOLUME 4, NuMBER 2, PP. 3-17. DOI: 10.35618/hsr2021.02.en003 
SaAti, S. - Tavana, M. - Hatami-Marbini, A. - Hajiakhondi, E. [2015]: A fuzzy linear programming model with fuzzy parameters and decision variables. International Journal of Information and Decision Sciences. Vol. 7. No. 4. pp. 312-333. https://doi.org/10.1504/ IJIDS.2015.074129

Villacorta, P. J. - Rabelo, C. A. - Pelta, D. A. - Verdegay, J. L. [2017]: FuzzyLP: An R Package for solving fuzzy linear programming problems. In: Kacprzyk, J. - Filev, D. Beliakov, G. (eds.): Granular, Soft and Fuzzy Approaches for Intelligent Systems. Studies in Fuzziness and Soft Computing. Vol. 344. Springer. Cham. pp. 209-230. https://doi.org/10.1007/978-3-319-40314-4_11

YAGER, R. R. [1978]: Ranking Fuzzy Subsets over the Unit Interval. 1978 IEEE Conference on Decision and Control including the $17^{\text {th }}$ Symposium on Adaptive Processes. IEEE (Institute of Electrical and Electronics Engineers). San Diego. pp. 1435-1437. https://doi.org/10.1109/CDC.1978.268154

ZimMERMANN, H. J. [1978]: Fuzzy programming and linear programming with several objective functions. Fuzzy Sets and Systems. Vol. 1. No. 1. pp. 45-55. https://doi.org/10.1016/01650114(78)90031-3 